\title{
Optimized Electron Column and Detection Scheme for Advanced Imaging and Analysis of Metals
}

\author{
David Wall $^{1}$, Fabian Cyril-Sasam ${ }^{1}$, Tomas Vystavel ${ }^{2}$ and Petr Wandrol ${ }^{2}$ \\ 1. FEI Company, Achtseweg Noord 5, 5651 GG, Eindhoven, The Netherlands. \\ 2. FEI Company, Podnikatelska 6, 61200 Brno, Czech Republic
}

In recent years, there has been a large growth in the development of detectors for FIB/SEM systems as the number of applications and techniques is growing. In-lens detectors have traditionally been optimized for imaging low take-off-angle back-scattered electrons with higher energies (low loss). This signal typically generates images which are high in materials contrast [1]. Conversely, below-lens detectors have the ability to capture much higher take-off-angle backscattered electrons due to their geometry [2]. Recent advances in detector segmentation with concentric rings have further allowed the separation of take-off angle signal without the need to change the working distance between the final lens and the sample. This higher take off angle delivers a strong channeling contrast in the specimen, while the highest take off angle reveals topographic information.

Advanced studies of metals and other polycrystalline samples demand the ability to easily identify different grain orientations and effects at grain boundaries. For 2-dimensional studies, the below-lens detector technology with concentric ring segmentation offers a perfect solution to separate all angles and detect topographic and channeling contrast in one image. However, DualBeam FIB/SEM systems are specifically designed to deliver site-specific sub-surface information and 3-dimensional volumes. However, this data is typically acquired with a tilted specimen, meaning that in most systems below-lens detectors cannot be used for these applications due to the possibility of collision between the sample and the detector.

In order to achieve stronger channeling contrast, a lower energy ( $2 \mathrm{keV}$ and below) is required due to reduced penetration depth and a higher sensitivity to the surface. In 3-dimensional studies this reduced energy and higher sensitivity enables thinner slicing and therefore better resolution.

A new electron column with a new detection scheme has been specifically developed to meet the needs for 2-D and 3-D studies of metals and other polycrystalline materials. The position of the BSE detector in the NICol ${ }^{\mathrm{TM}}$ SEM column makes it part of the final lens. This geometry enables it to detect a higher take-off-angle BSE signal [3], while not causing any issues for tilted specimen. This opens up the possibility for strong channelling contrast images to be gathered even while tilted, meaning investigation of grain boundaries, crack tips, and strain around nano-indentations can be performed easily in 3dimensions. A high signal to noise ratio is achieved both by position close to the sample surface, and also assisted by the presence of an electrostatic type lens which accelerates signal back into the detection system. This increased signal is essential to acquiring 3D volumes in a reduced time since image acquisition time is typically the main bottleneck in 3D workflows. Lastly, the in-lens BSE detector is split into two segments. By imaging with the sum of these two detector segments, a materials contrast image can be acquired, while subtracting these two segments, a topographical image can be generated. This high degree of flexibility from a single detector, together with other in-lens and in-chamber detector provides all the answers (topographic, materials and channelling contrast) that are required in a thorough investigation of a metal specimen. 


\section{References:}

[1] J. Cazaux et al, Ultramicroscopy 135 (2013) 43-49

[2] T. Aoyama et al, ISIJ International, vol. 51 (2011), No. 9, p. 1487-1491.

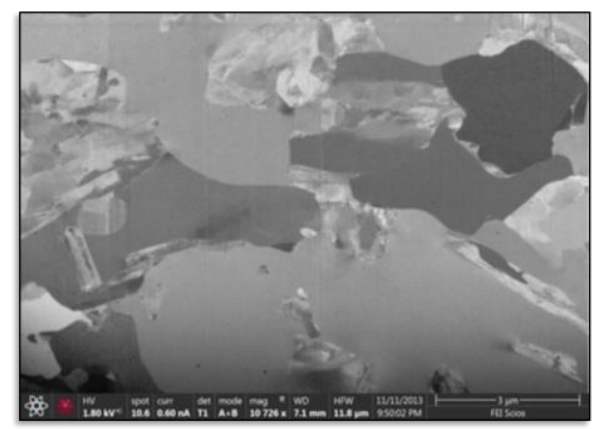

Figure 1. SEM image of FIB cross-section through steel sample acquired at $1.8 \mathrm{keV}$ exhibiting strong channeling contrast. This enables clear identification of austenite and ferrite regions.

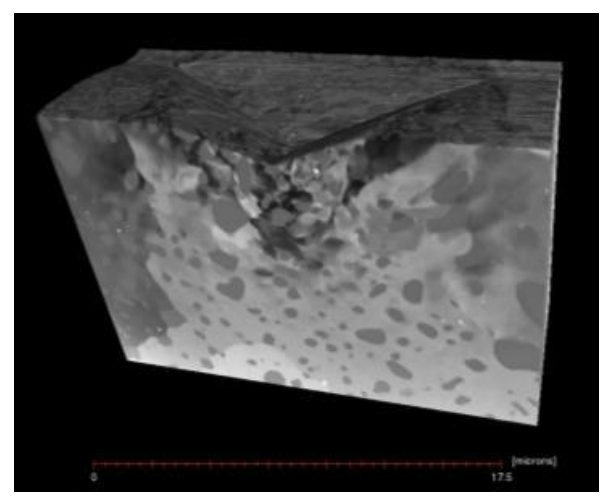

Figure 2. Volume rendering of a 3D stack of images acquired through a nano-indent in a steel specimen.

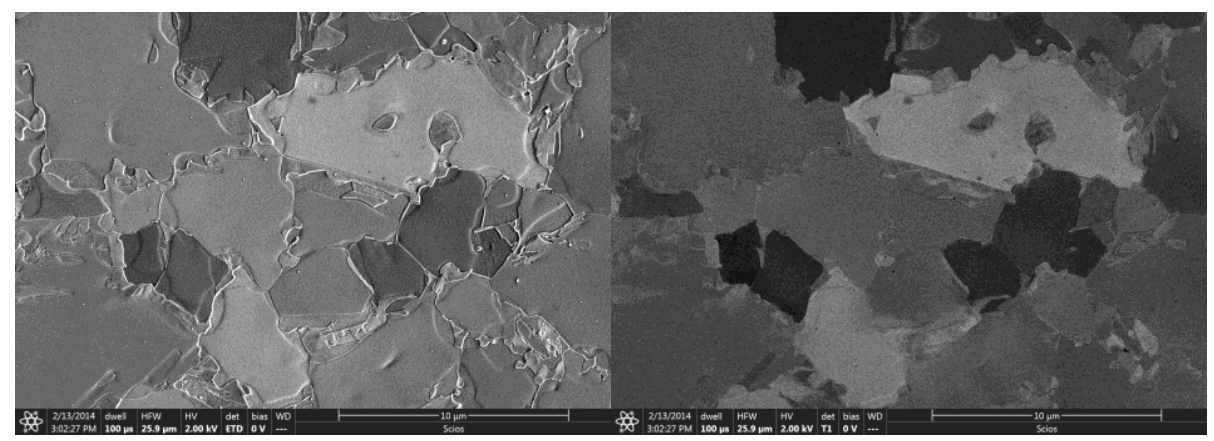

Figure 3. Simultaneous detection of a TRIP steel specimen using (left) low take-off-angle BSE's showing a high degree of topography, while high take-off-angle BSE's (right) show strong channeling information. Images are acquired at $2 \mathrm{keV}$. 